\section{MS9-P2 Structural view of fungal glutathionyl-hydroquinone reductases}

Claude DIDIERJEAN ${ }^{1,2}$, Mathieu Schwartz ${ }^{1}$, Thomas Perrot ${ }^{2}$, Arnaud Hecker ${ }^{2}$, Eric Gelhaye ${ }^{2}$, Frederique Favier ${ }^{1}$

1. University of Lorraine, UMR 7036 CRM2, Vandoeuvre-les-Nancy, France

2. CNRS, UMR 7036 CRM2, Vandoeuvre-les-Nancy, France

email: claude.didierjean@univ-lorraine.fr

Glutathione transferases (GSTs) constitute a widespread super-family of enzymes with highly specific to overlapping functions. These enzymes have been extensively studied in mammals because of their roles in xenobiotic conjugation. Despite this, GST functions are largely unexplored, especially in microorganisms. Our work focused on fungal glutathionyl-hydroquinone reductases (GHRs). These singular GSTs catalyze the reduction of hydroquinones-SG (Xun et al., 2010), and are present in all kingdoms but animals. The first GHR structure to be described in the saprotrophic fungus Phanerochaete chrysosporium (PcGHR1) has unveiled original features. An original dimerization mode led us to establish a new structural class named Xi (GSTX) (Meux et al., 2011).

Here we report the crystal structures of two GHRs, TvGHR1 from the basidiomycete Trametes versicolor and ScECM4 from the yeast Saccharomyces cerevisiae, at 2.28 and $1.45 \AA$ resolution, respectively. TvGHR1 and ScECM4 share the classical GST fold which consists in a thioredoxin $\mathrm{N}$-terminal domain and an all $\alpha \mathrm{C}$-terminal domain. Besides the features that have been uncovered in PcGHR 1 , additional secondary structures were found in the yeast ScECM4 structure. Like its characterized homologs, ScEcM4 is specific to the reduction of quinones conjugated with glutathione (Lallement et al., 2014). TvGHR1 is more polyvalent because it also reduces substrates rather accepted by Omega-class GSTs like phenylacetophenone-SG. Compared with known GHR structures, TvGHR1 exhibits a larger substrate binding site with an original motif that could explain the substrate versatility. Phylogenetic analyses revealed that fungal GHRs could be separated in two families : the 'classic' GHRs and the 'atypical' GHRs that exhibit motif variations as observed in TvGHR1. Furthermore, the conservation of GHRs in fungi suggests that they could fulfill a crucial role. Their diversification in wood-degrading basidiomycetes agrees with the hypothesis that GHRs would play a role in the metabolism of phenolic compounds (Belchik \& Xun, 2011).

Belchick S.M. \& Xun L., Drug Metab Rev. 2011, 43(2), 307 ; Lallement et al., FEBS Lett. 2015, 589, 37; Meux et al., J. Biol. Chem. 2011, 286(11), 9162; Xun et al., Biochem. J. 2010, 428(3), 419.

Keywords: glutathionyl-hydroquinone, reductase, fungi, glutathione transferase

\section{MS9-P3 PLA 2 s-like membrane perturbation mechanism: extracting the most of crystallography data} Rafael J. Borges ${ }^{1,2}$, Ney Lemke ${ }^{1}$, Massimo Sammito ${ }^{2}$, Claudia
Millán $^{2}$, Isabel Usón ${ }^{3}$, Marcos M.R. Fontes

1. Dept. of Physics and Biophysics, Biosciences Institute (IBB), São Paulo State University (UNESP), Botucatu, São Paulo, Brazil 2. Dept. of Structural Biology, Institute of Molecular Biology of Barcelona (IBMB), Spanish National Research Council (CSIC), Barcelona, Spain

3. Dept. of Structural Biology, Catalan Institution for Research and Advanced Studies (ICREA), Barcelona, Spain

email: rjborges@ibb.unesp.br

Venomics has been an invaluable tool to biomedicine by aiding comprehension of physiological mechanisms and drug design. Particularly, secreted phospholipases A $\left(\mathrm{PLA}_{2} \mathrm{~s}\right)$ had their catalytic mechanism first inferred evaluating three high resolution crystallographic structures PLA $\mathrm{s}$ from Chinese cobra and bee venom in different states ${ }^{2}$. A subtype of PLA $\mathrm{P}_{2}$, the $\mathrm{PLA}_{2} \mathrm{~s}$-like, also named as $\mathrm{PLA}_{2}$ s-homologue due to its similar tertiary structure and common ancestor, does not possess catalytic activity but still presents a high myotoxicity and a wide range of pharmacological activities 2,3 . The PLA $_{2}$ s-like versatility has related to its capability to perturb different biological membrane, breaking ionic homeostasis and inducing series physiological events that leads to cell death ${ }^{3}$. Although a lot has been discovered by functional and structural experiments, PLA s-like $_{2}$ exact mechanism of action is not fully understood ${ }^{2,3}$. Herein, we investigated their molecular mechanism by evaluation of global and local characteristics of crystallographic models. We gathered all snake venom $\mathrm{PLA}_{2}$ s-like structures available in Protein DataBank in the most probable dimer in solution using ProtCID database ${ }^{4}$. We evaluated models by hydrophobic channel accessibility through MOLE $2.0^{5}$, by presence or absence of hydrophobic ligands and by the geometrical orientation of one monomer to the other. The geometrical orientation was extracted from the rotation matrix after monomer superimposion using lsqkab program ${ }^{6}$ available in CCP4 suite ${ }^{7}$. By these global and local structural evaluations, we identified different native and complex states relating monomer-monomer geometry to hydrophobic channel aperture and closure.

\section{References}

1. Scott, D. L. et al. Science 250, 1541-1546 (1990). 2. Fernandes, C. A. H., et al. Borges, R. J., Lomonte, B. \& Fontes, M. R. M. Biochim. Biophys. Acta (2014). doi:10.1016/j.bbapap.2014.09.015 3. Lomonte, B., Angulo, Y., Sasa, M. \& Gutiérrez, J. M. Protein Pept. Lett. 16, 860-876 (2009). 4. Xu, Q. \& Dunbrack, R. L. Nucleic Acids Res. 39, D761-770 (2011). 5. Sehnal, D. et al. MOLE 2.0: advanced approach for analysis of biomacromolecular channels. J. Cheminformatics 5, 39 (2013). 6. Kabsch, W. Acta Crystallogr. Sect. A 32, 922-923 (1976). 7. Winn, M. D. et al. Acta Crystallogr. D Biol. Crystallogr. 67, 235-242 (2011).

Keywords: Phospholipase A2, geometry, hydrophobic channel 